\section{Strahlentherapie nach der Mastektomie: Tumorsubtyp beeinflusst die Effektivität}

\begin{abstract}
Brustkrebspatientinnen mit einem lokoregionären Rezidiv nach der Mastektomie können trotz schlechter Prognose von einer Radiotherapie profitieren. Der Tumorsubtyp korreliert dabei mit verschiedenen klinischen Parametern.
\end{abstract}

E ür ür die Klassifizierung von Tumorsubtypen eignen sich pathologische Marker wie Hormonrezeptor-, HER2-Status und Tumorgrading. So haben Patientinnen mit triple-negativem Mammakarzinom ein erhöhtes Risiko für lokoregionäre Rezidive (LRR) nach der Mastektomie. Die prognostische Bedeutung biologischer Tumorsubtypen in diesem Setting wurde bisher nicht umfassend charakterisiert.

Das Team um Yolanda D. Tseng evaluierte im Rahmen einer Studie das LRR-Risiko nach der Mastektomie vor dem Hintergrund moderner systemischer Therapien und den subtypspezifischen Effekt einer Post-Mastektomie-
Radiotherapie (PMRT) auf die LRRInzidenz.

Von 5.673 Patientinnen mit Brustkrebs im Stadium I-III, die sich einer Mastektomie unterzogen hatten, erhielten $30 \%$ eine PMRT. Die Forscher bildeten 5 Gruppen entsprechend dem Tumorsubtyp: Luminal A (ER/PR-positiv, HER2-negativ, niedriger/intermediärer Grad), Luminal B (ER/PR-positiv, HER2-negativ, hoher Grad), HER2-positiv mit bzw. ohne Trastuzumab-Behandlung und triple-negative Tumoren.

Binnen median 50,1 Monaten kam es zu 19 isolierten LRR (iLRR) und 109 LRREreignissen. Im Vergleich zur Gruppe mit einem Tumor vom Luminal-A-Typ war das iLRR-Risiko bei Patientinnen mit triple-negativem Subtyp signifikant erhöht (Hazard Ratio [HR] 14,10). Ein ähnlicher Trend zeichnete sich auch für den Luminal-B-Subtyp (HR 4,94) und HER2positive Patientinnen ohne TrastuzumabBehandlung ab (HR 4,41). Der protektive Effekt einer PMRT variierte zwischen den Untergruppen und war am ausgeprägtesten bei Patientinnen mit Luminal-A-Subtyp, am geringsten bei Frauen mit triplenegativen Tumoren.

Fazit: Mammakarzinompatientinnen mit einem triple-negativen Tumor profitieren nach einer Mastektomie am wenigsten von einer PMRT und entwickeln am häufigsten lokoregionäre Rezidive. Die Rolle lokaler Therapien für HER2positive Patientinnen sollte neu überdacht werden. Wolfgang Zimmermann

Tseng YD et al. Biological subtype predicts risk of locoregional recurrence after mastectomy and response to post-mastectomy radiation in a large, national database. Int J Radiat Oncol Biol Phys. 2015;93(3):622-30.

\section{Brustkrebs und Aromatasehemmer: Bisphosphonat besser von Anfang an?}

\author{
Aromataseinhibitoren können zur Abnahme der Knochendichte und so zu \\ einem erhöhten Frakturrisiko führen. Kann die Bisphosphonat-Gabe unter \\ Aromatasehemmer-Therapie den Knochenmasseverlust langfristig aufhalten?
}

D ie 1-Jahres-Daten der Studie N03CC hatten gezeigt, dass der sofortige Therapiebeginn mit Zoledronsäure den Knochenmasseverlust gegenüber einem verzögerten Beginn reduziert. Nun liegen die 5-Jahres-Ergebnisse vor. In der Phase-III-Studie erhielten 551 postmenopausale Frauen, die eine Therapie mit Tamoxifen beendet hatten und nun täglich Letrozol bekamen, randomisiert Zoledronsäure (4 mg i.v. alle 6 Monate) entweder von Beginn an oder erst, wenn der T-Score an Lendenwirbelsäule (LWS) oder Oberschenkelhals einen Wert von unter -2,0 erreicht hatte oder eine Fraktur aufgetreten war. Zusätzlich bekamen alle Frauen Kalzium und Vitamin D.

Der T-Score an der LWS war in der Gruppe mit sofortiger Zoledronsäure-
Therapie nach 5 Jahren insgesamt um 0,58 gestiegen und in der Gruppe mit verzögerter Therapie um 0,24 gesunken. Außerdem hatte die Knochendichte an der LWS nach 5 Jahren bei 10,2\% der Patientinnen mit sofortiger und bei $41,2 \%$ derer mit verzögerter Bisphosphonat-Therapie um $5 \%$ abgenommen ( $\mathrm{p}<0,0001)$.

2 Patientinnen mit sofortiger und 8 Patientinnen mit verzögerter Therapie entwickelten eine Osteoporose, allerdings war der Unterschied nicht signifikant ( $p=0,072)$. Auch bei der Anzahl an Frakturen gab es keinen signifikanten Unterschied (24 vs. $25 ; \mathrm{p}=0,84$ ).

Erhöhte Kreatininwerte (9 vs. $5 \%$ ) und Fieber ( 9 vs. $3 \%$ ) traten bei den Patientinnen mit Zoledronsäure-Therapie von Beginn an signifikant häufiger auf, zerebrovaskuläre Ischämien waren bei Patientinnen mit verzögerter Therapie häufiger. Hinsichtlich der häufigsten unerwünschten Ereignisse wie Arthralgie und Rückenschmerzen wurden keine signifikanten Unterschiede zwischen beiden Studienarmen beobachtet.

Fazit: Die sofortige Therapie mit Zoledronsäure schützte postmenopausale Frauen, die mit Letrozol behandelt wurden, langfristig besser vor einem Verlust an Knochenmasse als die verzögerte Gabe von Zoledronsäure. Allerdings waren die Inzidenzen von Osteoporose und Frakturen in den beiden Gruppen nicht signifikant unterschiedlich. Daher sprechen die Ergebnisse nicht nachhaltig für den routinemäßigen, sofortigen Beginn einer Therapie mit Zoledronsäure bei diesen Patientinnen. Judith Neumaier

Wagner-Johnston ND et al. 5-year follow-up of a randomized controlled trial of immediate versus delayed zoledronic acid for the prevention of bone loss in postmenopausal women with breast cancer starting letrozole after tamoxifen: N03CC (Alliance) trial. Cancer. 2015;121(15): 2537-43. 